\title{
Alimentos modificados e suas implicações no metabolismo energético
}

\section{Effects of modified foods on energy metabolism}

\section{RE S U M O}

Denise Machado MOURÃO'

Josefina Bressan Resende MONTEIRO2

Helen Hermana Miranda HERMSDORFF ${ }^{3}$

Marta Cristina Leite TEIXEIRA ${ }^{4}$

\section{Objetivo}

Comparar o efeito de duas dietas, modificadas no perfil de seus carboidratos, no metabolismo energético de homens com peso normal e com sobrepeso.

\section{Métodos}

Três formulações convencionais e suas formulações correspondentes diet, compuseram as dietas isoenergéticas padrão e a dieta modificada, respectivamente. Utilizou-se a calorimetria indireta, para obtenção dos dados referentes ao metabolismo energético. A amostra foi de 13 homens em cada grupo.

\section{Resultados}

O grupo com sobrepeso apresentou um gasto energético superior e uma termogênese inferior $(p<0,01)$ ao grupo com eutróficos, independentemente da dieta. Verificou-se um quociente respiratório de repouso, gasto energético de repouso, termogênese, e oxidação de carboidratos, superior $(p<0,05)$, após o uso de dieta modificada, independentemente do grupo.

\section{Conclusão}

Os resultados sugerem que uma dieta isoenergética, com maior quantidade de carboidratos complexos, tende a elevar o quociente respiratório, promovendo, assim, um aumento da termogênese e do gasto energético.

Termos de indexação: alimentos modificados, carboidratos complexos, metabolismo energético, obesidade.

\footnotetext{
$\overline{1}$ Doutoranda, Curso de Ciência e Tecnologia de Alimentos, Universidade Federal de Viçosa. Av. P.H. Rolfs, s/n, Campus da UFV, 36570-000, Viçosa, MG, Brasil. Correspondência para/Correspondence to: D.M. Mourão. E-mail: <dmourao@rocketmail.com>,<denisemm@vicosa.ufv.br>.

2 Departamento de Nutrição e Saúde, Universidade Federal de Viçosa. Viçosa, MG, Brasil.

${ }^{3}$ Mestranda, Curso de Nutrição, Universidade Federal de Viçosa. Viçosa, MG, Brasil.

${ }^{4}$ Mestranda, Curso de Microbiologia de Alimentos, Universidade Federal de Viçosa. Viçosa, MG, Brasil.
} 


\section{A B S T R A C T}

\section{Objective}

To compare the effects of two diets, which had their carbohydrates profile modified, on the energy metabolism of two groups of subjects: normal lean men and overweight ones.

\section{Methods}

Two isoenergetic meal plans were used, to evaluate their effects in the energy expenditure of thirteen lean, and thirteen overweight men. Three regular meal preparations and their diet analogous preparations were used to compound the regular meal and the modified meal, respectively. Indirect calorimetry was used to estimate the energy expenditure.

\section{Results}

Overweight subjects had a higher energy expenditure $(p<0.01)$ and lower thermogenesis $(p<0.01)$ comparing to lean subjects, independently of the meal tested. Modified meal was higher in complex carbohydrates and, independently of the group, its consumption caused rest respiratory quotient, rest energy expenditure, thremogenesis, and carbohydrate oxidation to be greater $(p<0.05)$ than those observed when regular meal was consumed.

\section{Conclusion}

Our results suggest that an isocaloric meal, higher in complex carbohydrate, can increase the respiratory coefficient, and, consequently, can increase thermogenesis and energy expenditure.

Indexing terms: modified food, complex carbohydrates, energy metabolism, obesity.

\section{N T R O D U Ç Ã O}

A obesidade é uma desordem complexa e multifatorial, caracterizada por ingestão energética acima do gasto, por um período prolongado. Tem sido descrita como desordem fisiológica que pode gerar complicações clínicas, psicológicas e sociais, com características de uma epidemia global'.

A oferta de novos produtos, com finalidades específicas para o combate à obesidade, tem sido cada vez mais comum no mercado; todavia, isso vem ocorrendo sem o conhecimento dos efeitos destes produtos no metabolismo energético. Assim, as mudanças na composição de macronutrientes em alimentos têm ocorrido de forma significativa, podendo afetar o balanço energético e a obesidade ${ }^{2}$. Em particular, tem-se verificado maior aumento no consumo de alimentos com alto teor de carboidratos refinados ${ }^{3}$.
Vários estudos têm investigado o efeito e a eficiência do uso de dietas hipocalóricas, alimentos modificados e outros, na obesidade ${ }^{4-6}$. Contudo, poucos trabalhos foram desenvolvidos para verificar o efeito desses alimentos e a sua eficiência no balanço energético de indivíduos obesos.

Metabolismo basal é a atividade metabólica necessária à manutenção da vida e das funções fisiológicas do indivíduo, ocorrendo, fundamentalmente, nos processos de transporte ativo, funções cardiorespiratórias, excreção, manutenção do tônus muscular, assim como nos processos de biossíntese das biomoléculas. O metabolismo basal é quantificado, medindo-se o consumo de oxigênio, a produção de $\mathrm{CO}_{2}$ e a excreção de nitrogênio da pessoa, tanto estando esta acordada, como quando em completo repouso, em atmosfera e temperatura neutras, após jejum noturno de 8 a 12 horas. A taxa metabólica basal, expressa por $\mathrm{kcal} / \mathrm{min} / \mathrm{kg}$ peso 
corporal, diferencia-se da taxa metabólica em repouso; enquanto aquela se refere ao metabolismo do organismo em jejum, a taxa metabólica de repouso, refere-se ao período pós-absortivo, incluindo gastos com a digestão, absorção e distribuição corporal do alimento ingerido. $\mathrm{Na}$ maioria dos adultos sedentários, o metabolismo basal constitui, aproximadamente, $60 \%$ a $70 \%$ dos gastos energéticos diários ${ }^{7}$.

A íntima relação entre o metabolismo basal e tamanho corporal é conhecida há muitos anos. Contudo, estudos recentes ${ }^{7}$ têm demonstrado que, para qualquer tamanho e composição corporal, o metabolismo basal pode variar consideravelmente entre os indivíduos. A massa livre de gordura, massa de gordura, idade e sexo, são os principais determinantes do metabolismo basal, explicando cerca de $80 \%$ de sua variação. Desta forma, a oxidação de substrato ocorre de maneiras diferentes nos vários tecidos corporais ${ }^{7}$.

Grande tem sido o uso de substitutos do açúcar na prevenção do ganho de peso. Tem-se verificado o aumento da oxidação de carboidratos e do gasto energético total, em resposta a uma sobrecarga de lipídios, ou seja, em resposta a mudanças agudas na composição da dieta ${ }^{8}$. Assim, verifica-se que alterações dietéticas podem, claramente, influenciar a regulação do peso corporal.

A calorimetria indireta tem sido um dos métodos mais utilizados para este tipo de estimativa, medindo o consumo de $\mathrm{O}_{2}$ e a produção de $\mathrm{CO}_{2}$ É utilizada na avaliação da termogênese e determinação da taxa de utilização de nutrientes ${ }^{9}$.

O presente trabalho teve o objetivo de investigar o efeito de uma dieta modificada (na constituição dos carboidratos) no metabolismo energético.

\section{CASUÍSTICA E MÉTODOS}

A realização deste estudo ocorreu no Laboratório de Composição Corporal e
Metabolismo Energético do Departamento de Nutrição e Saúde, na Divisão de Saúde e no Laboratório de Técnica Dietética do Departamento de Nutrição e Saúde, da Universidade Federal de Viçosa.

Vinte e seis homens saudáveis e não fumantes participaram do estudo. Exames laboratoriais de colesterol total e frações, glicose, triglicerídios, creatinina, uréia, ácido úrico e hemograma completo confirmaram o estado hígido dos voluntários. Estes foram divididos em dois grupos, o grupo de indivíduos eutróficos, com índice de massa corporal (IMC) entre 18,5 a $24,9 \mathrm{~kg} / \mathrm{m}^{2}$ e percentual de gordura corporal até $25 \%(n=13)$; e o grupo com sobrepeso, IMC e \% de gordura acima de $25^{1}(n=13)$. Para a avaliação do percentual de gordura, utilizou-se o método de bioimpedância elétrica.

Como o envelhecimento está associado às mudanças na composição corporal ${ }^{10}$, procurou-se trabalhar com voluntários cujas idades apresentassem uma variação mínima.

Todos os participantes assinaram o Termo de Consentimento Livre Esclarecido para a realização do estudo, previamente aprovado pelo Comitê de Ética na Pesquisa em Seres Humanos, da Universidade Federal de Viçosa.

Foi oferecido, como suprimento energético, um terço do gasto energético basal de cada indivíduo, sendo este também estimado pelo equipamento de bioimpedância elétrica.

Duas dietas isoenergéticas foram usadas, com aproximadamente $60 \%$ de carboidratos, $15 \%$ de proteína e $25 \%$ de lipídios: uma, como dieta padrão, utilizando-se alimentos preparados com sacarose; a outra, como dieta modificada, utilizando-se o adoçante sucralose, em substituiç̧ão à sacarose. Três preparações constituíram as dietas testadas: broa de milho, brigadeiro e doce de abacaxi.

Os percentuais de macronutrientes foram analisados pelo software DietPro ${ }^{\circledR}$ (versão 3.0), sendo que a dieta com sucralose apresentou maior quantidade de carboidratos complexos. 
Para o método da calorimetria indireta utilizou-se o aparelho Deltatrac $\|^{\circledR}$, que oferece medidas do consumo de oxigênio e produção de dióxido de carbono por minuto, através de um espirômetro. Obtivemos, então, os dados necessários para os cálculos posteriores de quociente respiratório (RQ). Este relaciona os moles de $\mathrm{CO}_{2}$ expirados por moles de $\mathrm{O}_{2}$ consumido; gasto energético (GE); termogênese induzida pela dieta (TID) e oxidação de nutrientes, lipídios e carboidratos. O RQ é calculado pela seguinte equação: $\mathrm{RQ}=\mathrm{VCO}_{2} \mathrm{NO}_{2}$.

Este foi, então, determinado para os respectivos períodos: jejum (RQJ), pós-prandial (RQPP) e de repouso (RQR). (1).

As medidas de volume de gás carbônico expirado $\left(\mathrm{VCO}_{2}\right)$ e o volume de oxigênio inspirado $\left(\mathrm{VO}_{2}\right) \mathrm{L}$ /minuto foram tomadas no tempo de 30 minutos, para o período de jejum (leituras de 5 em 5 minutos); 2 horas, para o período pós-prandial (com leituras de 15 em 15 minutos) e 30 minutos para o período de repouso (leituras de 5 em 5 minutos). Esta determinação é convertida em quilocalorias de calor produzido por metro quadrado de superfície corporal por hora, e é extrapolada para o gasto energético. O nitrogênio urinário foi determinado pelo método de Kjeldahl, descrito pela Association of Official Analytical Chemists $^{11}$, mediante a coleta da urina de cada voluntário, para os três períodos de medição do metabolismo energético: em jejum de $12 \mathrm{~h}$, após a refeição - pós prandial e no período de repouso.

O gasto energético ( $\mathrm{kcal} / \mathrm{min}$ ) é determinado pela seguinte equação:

$$
(\mathrm{kcal} / \mathrm{min})=\left(16,4 \mathrm{VO}_{2}\right)+\left(4,5 \mathrm{VCO}_{2}\right)-(3,4
$$
x N) / 4,18 (2).

O efeito das dietas na TID, para os períodos pós-prandial e de repouso, foi obtido por intermédio das seguintes equações:

1) $\operatorname{TIDPP}(\%)=($ GEPP - GEJ) $\times$ tempo (min) $\times 100 / \mathrm{kcal}$ ingeridas (3).

2) $\operatorname{TIDR}(\%)=($ GER - GEJ) $\times$ tempo (min) $\times 100 / \mathrm{kcal}$ ingeridas (4).
A quantidade de calor liberado no processo oxidativo não é constante, sendo dependente do tipo de nutriente que está sendo oxidado. $\mathrm{O} \mathrm{VO}_{2}$ e $\mathrm{VCO}_{2}$ consumido e produzido, respectivamente, permitem determinar com precisão a proporção dos diferentes substratos que estão sendo utilizados, assim como a energia produzida nesses processos. Para não haver variações no metabolismo, devido à dieta, utilizou-se uma mesma distribuição energética, em ambas as dietas testadas para os dois grupos.

Para os cálculos de oxidação de nutrientes, utilizou-se o quociente respiratório, o consumo de oxigênio e o dióxido de carbono, não protéicos, RQNP, $\mathrm{NPVO}_{2}$ e $\mathrm{NPVCO}_{2}$, respectivamente, $\mathrm{RQNP}=\mathrm{NPVCO}{ }_{2} / \mathrm{NPVO}_{2} \quad$ (5).

em que,

$$
\begin{aligned}
& { }^{a} \mathrm{PVO}_{2}=\mathrm{N} \times 6,25 \times 0,966 \\
& { }^{b} \mathrm{NPVO}_{2}=\mathrm{VO}_{2}{ }^{\mathrm{c}}-\mathrm{PVO}_{2} \\
& \mathrm{NPVCO}_{2}=\mathrm{VCO}_{2}-\mathrm{PVO}_{2} \\
& \mathrm{a}=\text { vol. } \mathrm{O}_{2} \text { consumido na oxidação protéica } \\
& \mathrm{b}=\text { vol. } \mathrm{O}_{2} \text { não-protéico } \\
& \mathrm{c}=\text { vol. } \mathrm{O}_{2} \text { total determinado por }
\end{aligned}
$$
calorimetria indireta

Oxidação de lipídio $(\mathrm{g} / \mathrm{min})=\mathrm{NPVO}_{2} \mathrm{x}$ (1 - RQNP)/0,293 x 0,746 (6).

Oxidação de carboidrato $(\mathrm{g} / \mathrm{min})=\mathrm{NPVO}_{2}$ x (RQNP - 0,707)/0,293 x 0,746 (7).

Todos os cálculos e as equações utilizadas foram baseados no trabalho de Labayen et al. ${ }^{9}$.

\section{Protocolo do teste}

Os indivíduos foram orientados a permanecer em jejum nas 12 horas que antecederam o início do teste, não realizando atividade física extenuante, e com o mínimo de esforço para chegar ao laboratório, sendo transportados de carro até o local. O protocolo utilizado para a avaliação do metabolismo energético está apresentado no Quadro 1. 
Quadro 1. Protocolo do teste para avaliação do metabolismo energético.

Chegada dos voluntários (jejum de $12 \mathrm{~h}$ e mínimo de atividade física)

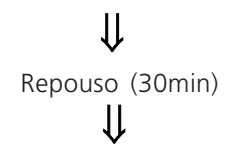

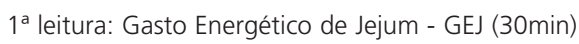

$\Downarrow$

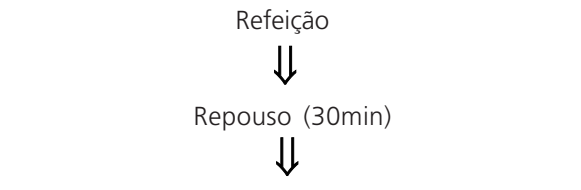

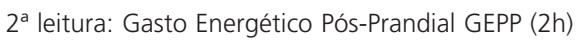

$\Downarrow$

Tempo livre com atividade sedentária ou leve (30min)

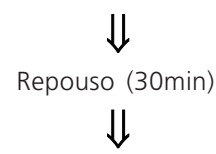

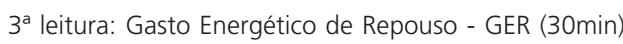

$7 \mathrm{~h}$

Coleta da urina referente

$7 \mathrm{~h} 30 \mathrm{~min}$ às $8 \mathrm{~h}$

$8 \mathrm{~h}$ às $8 \mathrm{~h} 30 \mathrm{~min}$

Coleta da urina

$9 \mathrm{~h}$ às $11 \mathrm{~h}$

Coleta da urina

$12 \mathrm{~h}$ às $12 \mathrm{~h} 30 \mathrm{~min}$
A temperatura ambiente foi registrada durante todo o teste, e apresentou média e erropadrão de $21,6 \pm 0,4^{\circ} \mathrm{C}$.

O experimento foi montado segundo esquema fatorial $2 \times 2$, tendo as duas dietas (com sacarose e com sucralose) e os dois grupos (o de indivíduos eutróficos e o dos com sobrepeso) como fatores, no delineamento em blocos casualisados com 13 repetições.

Os dados foram analisados por meio de análise de variância. As médias foram comparadas, utilizando-se o teste de " $F$ ", adotando-se o nível de $1 \%$ e $5 \%$ de probabilidade. Os dados foram analisados, utilizando-se o programa Sistema de Análises Estatística e Genéticas (SAEG).

\section{RES U LTA D O S}

Considerando a recomendação de consumo de um terço do gasto energético basal de cada indivíduo, o grupo de eutróficos consumiu cerca de $582 \pm 14,4 \mathrm{kcal}$ e o grupo com sobrepeso $673 \pm 14,8 \mathrm{kcal}$ (média \pm erro-padrão) para cada dieta. Não houve diferença $(p>0,05)$ entre o peso médio das dietas testadas dentro do mesmo grupo (dados não apresentados).

$\mathrm{Na}$ avaliação do efeito das duas dietas na resposta metabólica dos voluntários, verificou-se que a dieta com sucralose teve efeito superior $(p<0,05)$ para as variáveis RQR, GER, TIDPP, TIDR e OXID CHO. Para as variáveis RQPP, GEPP, e OXID LIP não se verificou diferença na resposta metabólica entre as dietas testadas $(p>0,05)$ (Tabela 1).

O perfil dos voluntários que participaram deste estudo constituiu-se da avaliação da idade, peso, altura, IMC, \% de gordura, gasto energético basal, com os valores médios e erro-padrão (Tabela 2).

A ANOVA não mostrou interação significativa entre os fatores grupo e dieta. Desta forma, procedeu-se ao estudo desses efeitos principais de forma independente.

Na comparação da resposta metabólica entre grupos, os gastos energéticos, tanto o de jejum e o pós-prandial, como o de repouso, foram superiores, enquanto que a termogênese pós-prandial e a de repouso, foram inferiores para 
os indivíduos com sobrepeso $(p<0,01)$. Contudo, não foi encontrada diferença significativa entre as médias do quociente respiratório entre os grupos $(p>0,01)$ (Tabela 2).

\section{I S C U S S Ã O}

\section{Efeito da manipulação dietética}

Dietas com diferentes fontes de carboidratos agem de forma diversa na oxidação de macronutrientes e, também, no gasto energético total ${ }^{2}$. A frutose tem uma velocidade de oxidação maior, dentre os monossacarídeos, por não depender da insulina; e, ainda, promove aumento na oxidação de carboidratos e redução na oxidação de lipídios ${ }^{12}$.

Estudos demonstraram um decréscimo no RQ de voluntários obesos, após o uso de dieta rica em lipídios e após 28 dias de intervenção ${ }^{13}$, com dieta hipocalórica, em obesos mórbidos hospitalizados $^{14}$.

Tabela 1. Valores médios das variáveis RQJ, RQPP, RQR, GEJ, GEPP, GER, TIDPP, TIDR, OXID LIP e OXID CHO para as dietas testadas (média e erro-padrão para $n=13$ ).

\begin{tabular}{|c|c|c|c|c|c|}
\hline & \multicolumn{2}{|c|}{ Sacarose } & \multicolumn{2}{|c|}{ Sucralose } & \multirow{2}{*}{ Valor de $p$} \\
\hline & M & $\mathrm{DP}$ & M & DP & \\
\hline Quociente respiratório pós-prandial (RQPP) & 0,85 & $0,01^{\mathrm{A}}$ & 0,84 & $0,01^{\mathrm{A}}$ & $(p>0,05)$ \\
\hline Quociente respiratório de repouso (RQR) & 0,81 & $0,01^{\mathrm{B}}$ & 0,84 & $0,01^{A}$ & $(p<0,05)$ \\
\hline Gasto energético de repouso (GER) (kcal/min) & 2213,19 & $\pm 51,21^{A}$ & 2260,23 & $\pm 43,54^{\mathrm{A}}$ & $(p>0,05)$ \\
\hline Gasto energético pós-prandial (GEPP) (kcal/min) & 2132,08 & $\pm 49,71^{\mathrm{B}}$ & 2282,81 & $\pm 40,32^{\mathrm{A}}$ & $(p<0,05)$ \\
\hline Termogênese pós-prandial (TIDPP) (\% Dieta) & 0,02 & $\pm 0,01^{B}$ & 0,03 & $0,01^{\mathrm{A}}$ & $(p<0,05)$ \\
\hline Termogênese de repouso (TIDR) (\% Dieta) & 0,05 & $0,01^{\mathrm{B}}$ & 0,09 & $0,01^{\mathrm{A}}$ & $(p<0,05)$ \\
\hline Oxidação de lipídios (OXIDLIP) (g/min) & 0,22 & $0,02^{\mathrm{A}}$ & 0,19 & $0,01^{\mathrm{A}}$ & $(p>0,05)$ \\
\hline Oxidação de carboidratos (OXIDCHO) (g/min) & 0,11 & $0,01^{\mathrm{B}}$ & 0,16 & $0,01^{A}$ & $(p<0,05)$ \\
\hline
\end{tabular}

As médias seguidas de uma mesma letra na linha não diferem entre si a $5 \%$ de probabilidade, pelo teste de $F$.

Tabela 2. Perfil geral dos voluntários e valores médios das variáveis RQJ, RQPP, RQR, GEJ, GEPP, GER, TIDPP, e TIDR, para os grupos estudados (média e erro-padrão para $\mathrm{n}=13$ ).

\begin{tabular}{|c|c|c|c|c|c|}
\hline & \multicolumn{2}{|c|}{ Eutróficos } & \multicolumn{2}{|c|}{ Sobrepeso } & \multirow{2}{*}{ Valor de $p$} \\
\hline & $\mathrm{M}$ & DP & M & DP & \\
\hline Idade (anos) & 25,00 & $\pm 1,60$ & 30,00 & $\pm 1,90$ & - \\
\hline Peso $(k g)$ & 69,20 & $\pm 1,60$ & 88,50 & $\pm 2,40$ & - \\
\hline Altura $(\mathrm{m})$ & 1,73 & $\pm \quad 0,01$ & 1,73 & 0,02 & - \\
\hline Índice de Massa Corporal ( $\left.\mathrm{kg} / \mathrm{m}^{2}\right)$ & 23,40 & $\pm 0,50$ & 29,50 & 0,60 & - \\
\hline Gordura Corporal Total (\%) & 15,60 & $\pm 0,50$ & 25,00 & 0,90 & - \\
\hline Quociente respiratório de jejum (RQJ) & 0,81 & $0,01^{\mathrm{A}}$ & 0,80 & $0,01^{\mathrm{A}}$ & $(p>0,01)$ \\
\hline Quociente respiratório pós-prandial (RQPP) & 0,85 & $0,01^{\mathrm{A}}$ & 0,84 & $0,01^{\mathrm{A}}$ & $(p>0,01)$ \\
\hline Quociente respiratório de repouso (RQR) & 0,83 & $\pm 0,01^{\mathrm{A}}$ & 0,82 & $0,01^{\mathrm{A}}$ & $(p>0,01)$ \\
\hline Gasto energético de jejum (GEJ) (kcal/min) & 1873,92 & $\pm 27,36^{\mathrm{B}}$ & 2138,77 & $\pm 38,44^{\mathrm{A}}$ & $(p<0,01)$ \\
\hline Gasto energético pós-prandial (GEPP) (kcal/min) & 2097,73 & $\pm 29,71^{\mathrm{B}}$ & 2375,69 & $\pm 46,20^{\mathrm{A}}$ & $(p<0,01)$ \\
\hline Gasto energético de repouso (GER) (kcal/min) & 2064,69 & $\pm 36,10^{B}$ & 2350,19 & $\pm 40,22^{A}$ & $(p<0,01)$ \\
\hline Termogênese pós-prandial (TIDPP) (\% Dieta) & 0,04 & $\pm 0,01^{\mathrm{A}}$ & 0,01 & $\pm 0,01^{B}$ & $(p<0,01)$ \\
\hline Termogênese de repouso (TIDR) (\% Dieta) & 0,09 & $0,01^{\mathrm{A}}$ & 0,06 & $0,01^{\mathrm{B}}$ & $(p<0,01)$ \\
\hline Oxidação de lipídios (OXIDLIP) (g/min) & 0,19 & $0,02^{\mathrm{A}}$ & 0,22 & $0,01^{\mathrm{A}}$ & $(p>0,01)$ \\
\hline Oxidação de carboidratos (OXIDCHO) (g/min) & 0,13 & $0,01^{\mathrm{A}}$ & 0,14 & $0,01^{\mathrm{A}}$ & $(p>0,01)$ \\
\hline
\end{tabular}

As médias seguidas de uma mesma letra na linha não diferem entre si a $1 \%$ de probabilidade, pelo teste de $F$. 
Ademais, tem-se verificado que, para dietas mistas, o valor de RQ está em torno de 0,8. A falta de significância no RQPP $(p>0,05)$ era, de certa forma, esperada (Tabela 1), pois este período pós-prandial, corresponde à digestão e à absorção das dietas, não sendo possível notar, muitas vezes, diferenças no RQ pelo efeito de uma dieta. Em dietas líquidas, alterações no RQ e, conseqüentemente, no gasto energético, são mais facilmente notadas ${ }^{15}$, pois os alimentos líquidos e com menor teor de lipídios são metabolizados mais rapidamente. Desta forma, decorridas três horas e meia após a ingestão das dietas testadas, com alimentos sólidos, foi possível observar maior RQR nos indivíduos do grupo com a dieta com sucralose, sugerindo que uma dieta isoenergética, com maior quantidade de carboidratos complexos, tende a produzir elevação no RQ e, conseqüentemente, na oxidação de carboidratos.

Embora diferenças no gasto energético tenham sido encontradas em vários estudos com macronutrientes ${ }^{16,17}$, Westerterp-Plantenga et al. ${ }^{18}$ consideram que a composição de uma refeição parece não influenciar no gasto energético.

Assim, como não houve diferença significativa entre as dietas testadas no RQPP, possivelmente devido ao pouco tempo de metabolização decorrido, o mesmo parece ter acontecido para o gasto energético pós-prandial. Já o gasto energético de repouso foi superior no grupo submetido à dieta com sucralose, resultado correlacionado a um maior valor da TIDPP e TIDR da mesma, visto que a termogênese é um dos componentes do gasto energético (Tabela 1). Entretanto, Raben et al. ${ }^{19}$ observaram maior gasto energético na dieta rica em carboidratos.

Não foi verificada nenhuma mudança significativa na oxidação de lipídios, após a ingestão das dietas testadas (Tabela 1), possivelmente porque ajustes, na oxidação de lipídios, relacionados à ingestão de alimentos, geralmente só ocorrem após um período de tempo considerável, acarretando mudanças da gordura corporal e subseqüentes mudanças na concentração de ácidos graxos circulante, uma vez que, para curtos períodos, a oxidação de lipídios depende, em maior escala, de fatores relacionados ao metabolismo de carboidratos, como o depósito de glicogênio ${ }^{12}$.

Com relação à oxidação de carboidratos, é necessário considerar que existem variações na absorção de diferentes tipos de carboidratos. Também os outros nutrientes presentes no alimento estão relacionados a estas variações, podendo, assim, refletir respostas termogênicas diferentes ${ }^{12}$.

Vários trabalhos têm relatado a superioridade da frutose para aumentar a TID e o gasto energético, quando comparada à glicose $\mathrm{e}^{12,20}$.

No trabalho de Blaak \& Saris ${ }^{12}$, diferentes tipos de carboidratos (frutose, sacarose, glicose e amido de milho) foram testados, em solução aquosa, para investigar possíveis mudanças no balanço de macronutrientes. Constatou-se incremento no gasto energético, sendo este similar entre frutose e sacarose, porém maior para sacarose, quando comparada à glicose. A TID foi similar, após a ingestão de frutose e sacarose, sendo maior com sacarose do que com glicose e amido, e maior para frutose do que para glicose. Aumentos na oxidação total de carboidratos foram significativamente maiores com sacarose do que com glicose e amido, concluindo-se que a termogênese e a utilização de substratos variam conside-ravelmente após a ingestão de diferentes tipos de carboidratos. Contudo, é importante mencionar que o amido utilizado nesse estudo era pré-gelatinizado, com boa digestibilidade (73\% de amilopectina e $27 \%$ de amilose), não se podendo assegurar que estes achados são válidos para outros tipos de amido de milho. De qualquer forma, esses resultados indicaram que a termogênese, após a ingestão de carboidratos, não é dependente do aumento da concentração de insulina plasmática, somente, mas resultante do metabolismo celular.

A dieta com sucralose provocou maior elevação $(p<0,05)$ na oxidação de carboidratos (Tabela 1). Outros estudos também mostraram diferenças neste tipo de oxidação, após a ingestão de carboidratos simples e complexos ${ }^{19,21,22}$. O 
trabalho de Ritz et al. ${ }^{22}$ mostrou maior oxidação de carboidratos para a dieta com carboidratos complexos, corroborando os resultados do presente trabalho. Contudo, Raben et al. ${ }^{19}$ observaram maior oxidação de carboidratos e menor oxidação de lipídios para a dieta com sacarose. Entretanto, esta dieta era superior em peso, implicando em maior ingestão energética. Vasilaras et al. ${ }^{23}$ não encontraram diferença significativa na oxidação de macronutrientes entre as dietas com carboidratos simples e complexos.

\section{Efeito da composição corporal}

Tem sido relatado que o gasto energético total tende a aumentar com o peso corporal ${ }^{24} \mathrm{e}$ que a relação entre o gasto e a massa livre de gordura é linear ${ }^{25}$. Contudo, sabe-se que a relação de tecido gorduroso/tecido magro está aumentada em obesos e que o tecido adiposo é metabolicamente menos ativo ${ }^{25}$. Um alto valor de $\mathrm{RQ}$ de $24 \mathrm{~h}$ tem sido considerado um fator de risco para o ganho de peso corporal ${ }^{26}$. Assim, altos valores de RQ, encontrados em indivíduos obesos, demonstram uma tendência para poupar gordura, favorecendo um aumento da oxidação de carboidratos, possivelmente por um defeito no metabolismo de oxidação de nutrientes, nos obesos $^{25}$. $\mathrm{O} \mathrm{VO} 2$ consumido, está diretamente relacionado ao tamanho corporal, enquanto que - $\mathrm{VCO}_{2}$ produzido, relaciona-se ao tipo de nutriente oxidado e à eficiência metabólica ${ }^{27}$.

Neste experimento não foi encontrada diferença significativa entre as médias de RQ na comparação entre os grupos (Tabela 2), possivelmente porque, neste estudo, comparou-se o metabolismo de indivíduos com peso normal e sobrepeso, e não obesos, não havendo assim uma magnitude significativa na gordura corporal total, a ponto de produzir um RQ diferente entre os grupos.

Já o gasto energético, nos períodos de jejum, pós-prandial e repouso, foi superior para o grupo com sobrepeso, caracterizado por uma maior superfície de área corporal total deste grupo, referente a um maior IMC (Tabela 2). Salienta-se aqui que, em geral, o gasto energético em indivíduos obesos é maior do que em magros ${ }^{25}$, mas a taxa metabólica, expressa pelo gasto energético por quilograma de peso corporal num determinado tempo, é menor.

Estudos complementares à calorimetria indireta também confirmaram maior gasto energético em obesos, quando comparados a magros do mesmo sexo e idade, utilizando-se o método de água duplamente marcada ${ }^{24}$.

Os valores inferiores da TIDPP e TIDR, em indivíduos com sobrepeso (Tabela 2), concordam com os resultados de outros trabalhos que também verificaram menor TID em obesos ${ }^{28,29}$, possivelmente, pela resistência insulínica, presente na grande maioria de obesos, podendo levar a uma menor eficiência metabólica dos mesmos.

Christin et al. ${ }^{30}$ demonstraram que a insulina, por si só, não estimula um aumento na termogênese, mas que esta estimulação é feita via sistema nervoso simpático, explicando o comportamento facultativo da TID.

Existem hipóteses recentes quanto aos possíveis "defeitos" na utilização de macronutrientes em indivíduos obesos ${ }^{25}$. Assim, esperava-se que a oxidação de lipídios fosse menor e a de carboidratos maior, no grupo com sobrepeso, denotando menor eficiência metabólica do obeso em oxidar gordura. Entretanto, não se encontrou esta diferença neste estudo $(p>0,05)$, possivelmente, devido ao valor de gordura corporal total do grupo com sobrepeso, cujos depósitos de gordura não teriam sido suficientemente grandes para determinar este efeito, como ocorre com obesos mais pesados.

Entretanto, McDevitt et al. ${ }^{2}$ não encontraram diferença significativa na oxidação de macronutrientes, mesmo entre mulheres magras e obesas, com diferenças grandes na composição corporal (IMC de 25 e $31 \mathrm{~kg} / \mathrm{m}^{2}$ e gordura corporal total de $35 \%$ e $45 \%$ ).

Em conclusão, no presente estudo, a dieta isoenergética, com maior quantidade de carboidra- 
tos complexos, elevou o quociente respiratório promovendo, assim, um aumento na termogênese e no gasto energético total. São necessários estudos de longo prazo do metabolismo energético, com uso contínuo desses alimentos, para nos esclarecer quanto às suas indicações na prática clínica.

\section{A GRADECIMENTOS}

Gostaríamos de agradecer a todos os voluntários, a sua colaboração, bem como à Fundação do Amparo a Pesquisa do Estado de Minas Gerais (FAPEMIG), e ao Conselho Nacional de Pesquisa (CNPq), o apoio financeiro concedido a este trabalho.

\section{REFER Ê NCIAS}

1. World Health Organization. Obesity: prevention and managing the global epidemic. Report of a WHO Consultation on obesity. Geneva; 1998.

2. McDevitt RM, Poppitt SD, Murgatroyd PR, Prentice AM. Macronutrient disposal during controlled overfeeding with glucose, fructose, sucrose, or fat in lean and obese women. Am J Clin Nutr. 2000; 72(2):369-77.

3. Drewnowski A, Popkin BM. The nutrition transition: new trends in the global diet. Nutr Rev. 1997; 55(2):231-43.

4. Agus MSD, Swain JF, Larson CL, Eckert EA, Ludwig DS. Dietary composition and physiologic adaptations to energy restriction. Am J Clin Nutr. 2000; 71(4):901-7.

5. Flechtner-Mors M, Alt A, Adler G, Ditschuneit HH, Jenkinson CP. Norepinephrine-induced glycerol release from adipose tissue: influence of age and body mass index in obese people. Nutrition. 2001; 17(9):729-31.

6. Stubbs RJ, Mullen S, Johnstone AM, Rist M, Kracht A, Reid C. How covert are covertly manipulated diets? Int J Obes Relat Metab Disord. 2001; 25(4):567-73.
7. Goran M. Energy metabolism and obesity. Med Clin North Am. 2000; 84(2):347-62.

8. Horton TJ, Drougas H, Brachey A, Reed GW, Peters JC, Hill JO. Fat and carbohydrate overfeeding in humans: different effects of energy storage. Am J Clin Nutr. 1995; 62(1):19-29.

9. Labayen I, Forga L, Martinez JA. Nutrient oxidation and metabolic rate as affected by meals containing different proportions of carbohydrate and fat, in healthy young women. Eur J Nutr. 1999; 38(3):158-66.

10. Hunter GR, Weinsier RL, Gower BA, Wetzstein C. Age-related decrease in resting energy expenditure in sedentary white women: effects of regional differences in lean and fat mass. Am J Clin Nutr. 2001; 73(2):333-7.

11. Association of Official Analytical Chemists. The official methods of analysis of the association of official analytical chemists. Washington, DC; 1984.

12. Blaak EE, Saris WHM. Postprandial thermogenesis and substrate utilization after ingestion of different dietary carbohydrates. Metabolism. 1996; 45(10):1235-42.

13. Astrup A, Buemann B, Western P, Toubro S, Raben A, Christensen NJ. Obesity as an adaptation to a high-fat diet: evidence from a cross-sectional study. Am J Clin Nutr. 1994; 59(2):350-5.

14. Valtuena S, Sola R, Salas-Salvado J. A study of the prognostic respiratory markers of sustained weight loss in obese subjects after 28 days on VLCD. Int J Obes Relat Metab Disord. 1997; 21(4):267-73.

15. Habas ME, Macdonald. Metabolic and cardiovascular responses to liquid and solid test meals. Br J Nutr. 1998; 79:241-7.

16. Roberts SB, Fuss P, Beyman MB, Young VR. Influence of age on energy requirements. Am J Clin Nutr. 1995; 62:1053-8.

17. Cooling J, Blundell J. Differences in energy expenditure and substrate oxidation between habitual high fat and low fat consumers (phenotypes). Int J Obes Relat Metab Disord. 1998; 22(7):612-8. 
18. Westerterp-Platenga MS, Wijckmans-Duysens NA, Verboeket-van de Venne WPHG, de Graaf K, Westrate JA, van het Hof KH. Diet-induced thermogenesis and satiety in humans after full-fat and reduced-fat meals. Phys Behav. 1997; $61(2): 343-9$.

19. Raben A, MacDonald I, Astrup A. Replacement of dietary fat by sucrose or starch: effects of $14 \mathrm{~d}$ ad libitum energy intake, energy expenditure and body weight in formerly obese and never-obese subjects. Int J Obes Relat Metab Disord. 1997; 21(10):846-59.

20. Schwarz J, Schutz Y, Piolino V, Schneider H, Felber J, Jequier E. Thermogenesis in obese women: effect of fructose vs. glucose added to a meal. Am J Physiol. 1992; 262(4 Pt 1):E394-401.

21. Raben A, Andersen Helene B, Christensen Niels J, Madsen J, Holst Jens J, Astrup A. Evidence for an abnormal postprandial response to a high-fat meal in women predisposed to obesity. Am J Physiol. 1994; 267(4 Pt1):E549-E59.

22. Ritz P, Krempf M, Cloarec D, Champ M, Charbonnel B. Comparative continous-indirect-calorimetry study of two carbohydrates with different glycemic indices. Am J Clin Nutr. 1991; 54(4):855-9.

23. Vasilars TH, Raben A, Astrup A. Twenty-four hours energy expenditure and substrate oxidation before and after 6 months' ad libitum intake of a diet rich in simple or complex carbohydrates or a habitual diet. Int J Obes Relat Metab Disord. 2001; 25(7):954-65.
24. Prentice AM, Black AE, Coward WA, Cole TJ. Energy expenditure in overweight and obese adults in affluent societies: an analysis of 319 doubly-labeled water measurements. Eur J Clin Nutr. 1996; 50(2):93-7

25. Mela DJ, Rogers PJ. Food, eating and obesity. London: Chapman \& Hall; 1998.

26. Zurlo F, Lillioja S, Puente AED, Nyomba BL, Raz I, Saad MF, et al. Low ratio of fat to carbohydrate oxidation as predictor of weight gain study of 24-H Rq. Am J Physiol. 1991; 259(5 Pt 1):E650-E7.

27. Rosado EL. Estudo da composição corporal e do metabolismo energético em mulheres normais, obesas e pós-obesas estáveis. Viçosa: Universidade Federal de Viçosa; 1999.

28. Seidell JC, Muller DC, Sorkin JD, Andres R. Fasting respiratory exchange ratio and resting metabolic rate as predictors of weight gain: the baltimore longitudinal study on aging. Int J Obes Relat Metab Disord. 1992; 16(9):667-74.

29. Dabbech M, Boulier A, Apfelbaum MD, Aubert R. Thermic effect of meal and fat mass in lean and obese men. Nutr Res. 1996; 16(7):1133-41.

30. Christin L, Nacht CA, Vernet O, Ravussin E, Jequier $E$, Acheson KJ. Insulin: its role in the thermic effect of glucose. J Clin Invest. 1986; 77(6):1747-55.

Recebido para publicação em 29 de agosto de 2003 e aceito em 7 de abril de 2004. 